\title{
Efecto del Recubrimiento Comestible en las Propiedades de Trozos de Batata (Ipomoea Batatas Lam) Fritos por Inmersión. Parte 2: Propiedades Termofísicas y de Transporte
}

\author{
Armando Alvis*, Adriana González y Guillermo Arrázola \\ Universidad de Córdoba. Programa de Ingeniería de Alimentos. Facultad de Ingenierías. Grupo \\ Investigación Procesos y Agroindustria de vegetales. Carrera 6 N 76-103, Córdoba, Colombia. \\ (e-mail: aalvis2@hotmail.com) \\ ${ }^{*}$ Autor a quien debe ser dirigida la correspondencia
}

Recibido Jun. 9, 2014; Aceptado Ago. 20, 2014; Versión final recibida Sep. 1, 2014

\begin{abstract}
Resumen
Se estudió el efecto de la carboximetilcelulosa y la temperatura en las propiedades termofísicas, los coeficientes de transferencia de calor y masa, pérdida de humedad y ganancia de aceite durante la fritura de trozos de batata. El calor específico, conductividad térmica y densidad se determinaron mediante ecuaciones experimentales y la difusividad térmica se determinó según su definición física a partir de los valores de las otras propiedades termofísicas. Los coeficientes de transferencia de calor y masa se determinaron gráficamente basados en las soluciones de las ecuaciones diferenciales de conducción de calor y de concentración de humedad, en una dimensión y utilizando la regla de la superposición para ajustarla a un plato finito. Los resultados indicaron que las propiedades termofísicas de la batata varían significativamente durante la etapa de freído. El coeficiente de transferencia de calor $h$ es mayor cuando aumenta la temperatura del aceite y se utiliza recubrimiento. El coeficiente de transferencia de masa kc es mayor cuando aumenta la temperatura del aceite y no se utiliza recubrimiento comestible. Además, se observó que el recubrimiento comestible disminuye más del $30 \%$ el contenido de aceite final para todas las temperaturas de freído utilizadas, lo que hace que sea una excelente elección para mejorar la calidad nutricional de los productos fritos.
\end{abstract}

Palabras clave: Carboximetilcelulosa, propiedades termofísicas, coeficiente de transferencia de calor y masa, perdida de humedad

\section{Effect of Edible Coating on the Properties of Sweet Potato Slices (Ipomoea Batatas Lam) Cooked by Deep-Fat Frying. Part 2: Thermophysical and Transport Properties}

\begin{abstract}
The effect of temperature on Carboxymethylcellulose and thermophysical properties, heat transfer coefficients and mass, moisture loss and oil uptake during frying of potato chips was studied. The specific heat, thermal conductivity and density were determined using experimental equations and the thermal diffusivity was determined by their physical definition from the values of other thermophysical properties. The heat transfer coefficients and mass were determined graphically based on the solutions of the differential equations of heat conduction and high moisture, and using one-dimensional overlap rule to conform to a finite plate. The results indicated that the thermophysical properties of sweet potatoes vary significantly during the frying stage. The heat transfer coefficient $\mathrm{h}$ is greater when the oil temperature increases and coating used. The mass transfer coefficient $\mathrm{kc}$ is greater as the temperature increases and no edible oil is used. Moreover, the edible coating decreases more than $30 \%$ oil content for all end frying temperatures used, which makes it an excellent choice for improving the nutritional quality of the fried products.
\end{abstract}

Keywords: carboxymethylcellulose, thermophysical properties, heat transfer coefficient, mass transfer coefficient, moisture loss. 


\section{INTRODUCCIÓN}

Las propiedades termofísicas dan cuenta de las condiciones en que se desarrolla la transferencia de calor entre el fluido caliente y el alimento durante el proceso de fritura (Vitrac et al., 2002; Alvis et al., 2009a). Son parámetros esenciales en el modelamiento de varios procesos térmicos y en el diseño de sistemas de calentamiento. También, son importantes en la caracterización de procesos de transferencia de calor en la industria alimentaria, donde las principales consideraciones son los costos energéticos, la calidad y la seguridad del alimento (Hassan y Ramaswamy, 2011).

La conductividad térmica $k$ es una medida de la velocidad a la cual el calor es conducido en un material, es un coeficiente que cuantifica la velocidad de transferencia de calor en estado estable dQ/dt a lo largo de la dirección de un gradiente de temperatura dT/dt, gobernada por la ley de Fourier de conducción de calor. La difusividad térmica $\alpha$ es la relación de la conductividad térmica con la capacidad calórica de un material y es igual a $k / \rho C p$; este parámetro cuantifica la habilidad de un material para almacenar energía térmica durante un proceso de transferencia de calor. La difusividad térmica también se puede definir como la velocidad a la cual la energía térmica se difunde por conducción a través del material, es la propiedad térmica controlante durante el proceso de calentamiento conductivo transitorio (Huang y Liu, 2009). El calor específico de un material es la cantidad de calor necesaria para aumentar su temperatura en una unidad por unidad de masa, sin cambiar de estado (Valiente, 2001). El modelo matemático más utilizado para conocer las propiedades termofísicas es el desarrollado por Choi y Okos (1985) basado en la temperatura, en un rango de $-40^{\circ} \mathrm{C}$ a $150^{\circ} \mathrm{C}$, y la composición que tiene el alimento de humedad, proteína, grasa, fibra, carbohidratos y cenizas (Sablani, 2005; Farinu y Baik, 2007a).

En el área de la ingeniería, existen parámetros esenciales para la investigación termodinámica y el modelamiento de los tratamientos térmicos porque, en gran medida, son éstos los que determinan la velocidad de los procesos de transferencia de calor y de masa. Entre estos parámetros se encuentran los coeficientes de transferencia de calor y de masa (Farinu y Baik, 2007a). La interacción de los fenómenos de transferencia de calor y masa hace que la fritura sea una de las operaciones unitarias más compleja.

La transferencia de calor es un proceso donde la energía transita de un cuerpo a otro debido a una diferencia de temperaturas entre éstos. El coeficiente de transferencia de calor $h$ es una constante de proporcionalidad entre el flujo de calor y la diferencia de temperaturas entre la superficie de la muestra y el fluido (Farinu y Baik, 2007b); se define como la tasa de calor que puede ser transmitido por convección en la interacción entre la superficie del producto y el fluido, a través de un área superficial unitaria y un gradiente de temperatura unitario y se expresa en unidades internacionales de $W / m^{2}{ }^{\circ} \mathrm{C}$ (Alvis et al., 2009b). Durante el proceso de freído por inmersión la transferencia de calor ocurre de dos formas: por convección entre la superficie del alimento y el aceite caliente y por conducción dentro del alimento (Farkas et al., 1996a). El coeficiente de transferencia de calor depende de las condiciones en la capa límite, en las que influyen la geometría de la superficie y la naturaleza del movimiento del fluido (Incropera y DeWitt, 1999). Durante el freído, influyen las características físicas y químicas del aceite y del alimento, los parámetros del proceso como la temperatura y el tiempo de calentamiento (Alvis et al., 2009b). Existen varios autores que han reportado métodos diferentes para determinar $h$ (Farkas et al., 1996a, 1996b; Sahin et al., 1999; Vitrac et al., 2002; Budžaki y Šeruga; 2005; Sosa-Morales et al., 2006; Yildiz et al., 2007; Farinu y Baik, 2007b, 2008; Alvis et al., 2009a; Erdogdu y Dejmek, 2010; Kim et al., 2011).

La transferencia de masa es un proceso que se caracteriza porque hay un flujo de una sustancia que pasa a través de otras a escala molecular debido a un gradiente de concentración, con una rapidez que depende de la naturaleza del movimiento del fluido y de las propiedades fisicoquímicas del fluido y de la muestra. El coeficiente de transferencia de masa $k c$ es una constante que indica la velocidad de transferencia de una sustancia que atraviesa cierta superficie (Treybal, 2002). Durante el freído, la humedad del alimento se transfiere en forma líquida desde el centro hasta el frente de evaporación por capilaridad y luego el vapor se difunde a través de la estructura porosa de la costra para eliminarse al aceite y finalmente al medio ambiente (Farinu y Baik, 2005). El coeficiente de difusión $D$ es una medida de la movilidad de difusión de un componente o sustancia en un medio y se define como la relación entre el flujo de una cantidad de dicha sustancia por unidad de área y su gradiente de concentración. Es una propiedad del sistema que depende de la temperatura, presión y la naturaleza de los componentes. Su dimensión es longitud²/tiempo (Treybal, 2002). Varios autores han reportado métodos para calcular $D$ y kc (Farkas et al., 1996a, 1996b; Vitrac et al., 2002; Yamsaengsung y Moreira, 2002; Sosa-Morales et al., 2006; Yildiz et al., 2007; Farinu y Baik, 2008; Alvis et al., 2009a; Mosavian y Karizaki, 2012).

El objetivo de este estudio fue analizar el efecto que tienen el recubrimiento comestible y la temperatura en las propiedades termofísicas, los coeficientes de transferencia de calor y de masa y la pérdida de humedad y la ganancia de aceite durante el freído por inmersión de trozos de batata. 


\section{MATERIALES Y MÉTODOS}

\section{Preparación de la muestra}

Las unidades de batata se cortaron manualmente con las dimensiones de $1 \mathrm{~cm} \times 1 \mathrm{~cm} \times 5 \mathrm{~cm}$. Todas las muestras fueron sometidas a escaldado en una solución acuosa al $0.5 \%$ de cloruro de calcio en ebullición durante 5 minutos y se secaron con papel absorbente por un minuto. La mitad de las muestras fueron sometidas al pretratamiento de recubrimiento comestible y la otra mitad fue sometida a freído directamente. El pretratamiento de recubrimiento comestible se realizó sumergiendo las muestras en una solución acuosa de carboximetil celulosa $\mathrm{CMC}$ al $0.5 \%$ a temperatura ambiente durante un minuto. Se dejaron en escurridor metálico mientras se secaban con un secador manual con velocidad de $28.53 \mathrm{mph}$ ó $12.75 \mathrm{~m} / \mathrm{s} \mathrm{y}$ temperatura aproximada de $98^{\circ} \mathrm{C}$ durante seis minutos. El proceso de freído se realizó con aceite de palma en una freidora a tres temperaturas: 150,170 y $190^{\circ} \mathrm{C}$, y dos pretratamientos: escaldado y escaldado más recubrimiento comestible.

\section{Propiedades termofísicas}

Las propiedades termofísicas de conductividad $k$, calor específico $C p$ y densidad $\rho$, fueron determinadas con base en el contenido de humedad y la temperatura de freído utilizando las fórmulas sugeridas por Farinu y Baik (2008) para determinar las propiedades termofísicas de la batata durante el freído por inmersión relacionadas en la tabla 1. La difusividad térmica $\alpha$ se determinó según la relación $\alpha=k /(\rho C p)$, a partir de los valores obtenidos según las correlaciones presentadas en la tabla 1 . Donde $T$ es la temperatura en ${ }^{\circ} \mathrm{C}$ y $m$ es la fracción másica en base húmeda.

Tabla 1. Modelos de propiedades termofísicas para batata durante freído (Farinu y Baik, 2008)

\begin{tabular}{|c|c|c|c|}
\hline Propiedades & $\begin{array}{c}\text { Modelo/ Técnica } \\
\text { experimental }\end{array}$ & Unidades & Modelos \\
\hline Conductividad térmica & Farinu y Baik (2007a) & $\mathrm{W} / \mathrm{m} \mathrm{K}$ & $\mathrm{k}=0,1613+0,0014 \mathrm{~T}+0,2924 \mathrm{~m}+3,4839 \times 10^{-7} \mathrm{~T}^{2}$ \\
\hline Calor específico & $\begin{array}{c}\text { Método DSC entre } 20 \\
\text { y180 o } \text {-C humedad } \\
\text { de } 0.4-0.75 \text { bh }\end{array}$ & $\mathrm{kJ} / \mathrm{kg} \mathrm{K}$ & $\mathrm{Cp}=489,8+3313 \mathrm{~m}+24 \mathrm{~T}-53 \mathrm{mT}+33,7 \mathrm{~m}^{2} \mathrm{~T}$ \\
\hline Densidad & Multipicnómetro & $\mathrm{Kg} / \mathrm{m}^{3}$ & $\rho=1553-568,8 \mathrm{~m}$ \\
\hline
\end{tabular}

Propiedades de transporte: coeficientes $h$ y kc

Se determinó el valor del coeficiente efectivo de transferencia de calor gráficamente según la metodología reportada de Yildiz et al. (2007), Alvis et al. (2009a) y Kim et al. (2011), mediante ecuación (1):

$\ln \left(\frac{T(x, y, t)-T_{\infty}}{T_{i}-T_{\infty}}\right)=\ln A-2 \mu_{1}^{2} \frac{\alpha t}{L^{2}}$

$A=\left(\frac{2 \operatorname{sen} \mu_{1}}{\mu_{1}+\left(\operatorname{sen} \mu_{1}\right)\left(\cos \mu_{1}\right)}\right)^{2} \cos \left(\mu_{1} \frac{x}{L}\right) \cos \left(\mu_{1} \frac{y}{L}\right)$

Graficando y ajustando a una línea recta se obtiene el valor de la raíz $\mu_{1}$. Este valor fue utilizado para calcular el número de Biot y el coeficiente de transferencia de calor con las ecuaciones 3 y 4 , respectivamente.

$B i=\mu_{1} \operatorname{Tan} \mu_{1}$

$h=\frac{B i k}{L}$

Donde $T(x, y, t)$ en ${ }^{\circ} C$ es la temperatura en el centro de alimento en el tiempo $t$ en $s$, $T_{\infty}$ en ${ }^{\circ} C$ es la temperatura del medio de calentamiento, $\mathrm{Ti}$ en ${ }^{\circ} \mathrm{C}$ es la temperatura inicial del alimento, $\alpha$ es la difusividad térmica en $\mathrm{m}^{2} / \mathrm{s}$, $k$ es la conductividad térmica en $W / m{ }^{\circ} C, h$ es el coeficiente de transferencia de calor en $W / m^{2}{ }^{\circ} C, x$ y $y$ son las dimensiones de la muestra, $L$ es la mitad del espesor de la muestra en $m$, Bi es el número de Biot de transferencia de calor, y $\mu_{1}$ es la primera raíz de la ecuación característica. 
Se determinó el valor del coeficiente efectivo de transferencia de masa gráficamente según la metodología reportada de Yildiz et al. (2007) y Alvis et al. (2009a), conociendo la concentración promedio de humedad en el tiempo, según la ecuación 5:

$$
\begin{aligned}
& \ln \left(\frac{\bar{C}(t)-C_{\infty}}{C_{i}-C_{\infty}}\right)=2 \ln E-2 \mu_{1}^{2} \frac{D t}{L^{2}} \\
& E=\frac{2 \operatorname{sen}^{2} \mu_{1}}{\mu_{1}\left[\mu_{1}+\left(\operatorname{sen} \mu_{1}\right)\left(\cos \mu_{1}\right)\right]}
\end{aligned}
$$

Mediante la gráfica se obtuvo del intercepto el valor de $\mu_{1}$ y de la pendiente el valor de la difusividad efectiva de la humedad $D$, según las ecuaciones 5 y 6 . Estos valores se utilizaron para calcular el número de Biot de transferencia de masa y el coeficiente efectivo de transferencia de masa ecuaciones 7 y 8 , respectivamente.

$$
\begin{aligned}
& B i_{m}=\mu_{1} \operatorname{Tan} \mu_{1} \\
& k_{C}=\frac{B i_{m} D}{L}
\end{aligned}
$$

Donde $\bar{C}(t)$ en $\mathrm{kg}$ humedad/kg sólidos es la concentración promedio de humedad en el tiempo $t$ en $s$, $C_{\infty}$ es la concentración de humedad del medio de calentamiento, $\mathrm{Ci}$ es la concentración de humedad inicial del alimento en $\mathrm{kg}$ humedad/kg sólidos, $D$ es la difusividad de humedad en $\mathrm{m}^{2} / \mathrm{s}, k_{C}$ es el coeficiente de transferencia de masa en $\mathrm{m} / \mathrm{s}$, $L$ es la mitad del espesor de la muestra en $m, B i_{m}$ es el número de Biot de transferencia de masa, y $\mu_{1}$ es la primera raíz de la ecuación característica. El contenido de humedad y de grasa fue determinado mediante los respectivos métodos de la AOAC (2003) en diferentes tiempos de freído que permitieron obtener un gráfico representativo. Para cada una de las temperaturas, los tiempos fueron los siguientes:

$150 \stackrel{\circ}{ } \mathrm{C}: 0,15,30,40,60,120,180,240,300,360$ y 420 segundos.

$170 \stackrel{\circ}{\circ} \mathrm{C}: 0,15,30,40,60,120,180,240$ y 300 segundos.

$190 \stackrel{\circ}{\circ} \mathrm{C}: 0,15,30,40,60,120,180,240$ y 300 segundos.

Donde los tiempos cero ( 0 ) segundos indican las condiciones iniciales de las muestras justo antes de sumergirlas en el aceite. Las muestras, al sacarse del aceite, se pusieron sobre papel absorbente por 30 segundos para remover el aceite superficial y así evitar la alteración en el contenido de aceite absorbido en el período de enfriamiento. Luego de cada freído, el nivel del aceite fue revisado y repuesto a la freidora hasta el nivel inicial. Para analizar estadísticamente la influencia de la temperatura y el recubrimiento sobre las propiedades termofísicas, $h$ y $k c$ y sobre la absorción de aceite y pérdida de humedad, se aplicó un diseño de experimentos factorial en bloques, donde se consideró el tiempo de fritura como el factor de bloqueo y se tomaron como tratamientos las seis combinaciones de los factores temperatura y recubrimiento, cuyos niveles fueron 150,170 y $190 \stackrel{\circ}{C}$ para la temperatura y ausencia (0) y presencia $(0.5)$ para la cobertura. El número de repeticiones por tratamiento fueron tres.

\section{RESULTADOS Y DISCUSIÓN}

\section{Propiedades Termofísicas}

En esta investigación se calcularon las propiedades termofísicas de la batata durante diferentes tiempos de freído para cada uno de los tratamientos. Los valores para $C p, k, \alpha$ y $\rho$ se reportan en la figura 1 para todos los tratamientos.

El resultado del análisis de varianza para las propiedades termofísicas indicó que en el calor específico, conductividad y difusividad térmica hay un efecto altamente significativo del factor de bloqueo, de la temperatura y del recubrimiento, pero no se encontró efecto de la interacción de los factores temperatura y recubrimiento. Mediante una prueba de Tukey se encontraron que todos las diferencias fueron significativas, teniendo que, para las muestras con recubrimiento los valores de $\mathrm{Cp}, \mathrm{k}$ y a fueron mayores que para las muestras sin recubrimiento. El mismo comportamiento lo tuvieron las muestras que fueron fritas a menor temperatura; es decir, las muestras fritas a $150^{\circ} \mathrm{C}$ tuvieron valores mayores de $\mathrm{Cp}, \mathrm{k}$ y $\alpha$ que las fritas a 170 C, y éstas a su vez que las de $190 \stackrel{\circ}{\circ}$.

Con respecto a la densidad, el resultado del análisis de varianza indicó que hay un efecto altamente significativo del factor de bloqueo, de la temperatura y del recubrimiento, pero no se encontró efecto de la interacción de los factores temperatura y recubrimiento. Mediante una prueba de Tukey se encontró que todas las diferencias fueron significativas, teniendo para las muestras sin recubrimiento los valores $\rho$ fueron mayores que las muestras con recubrimiento. 

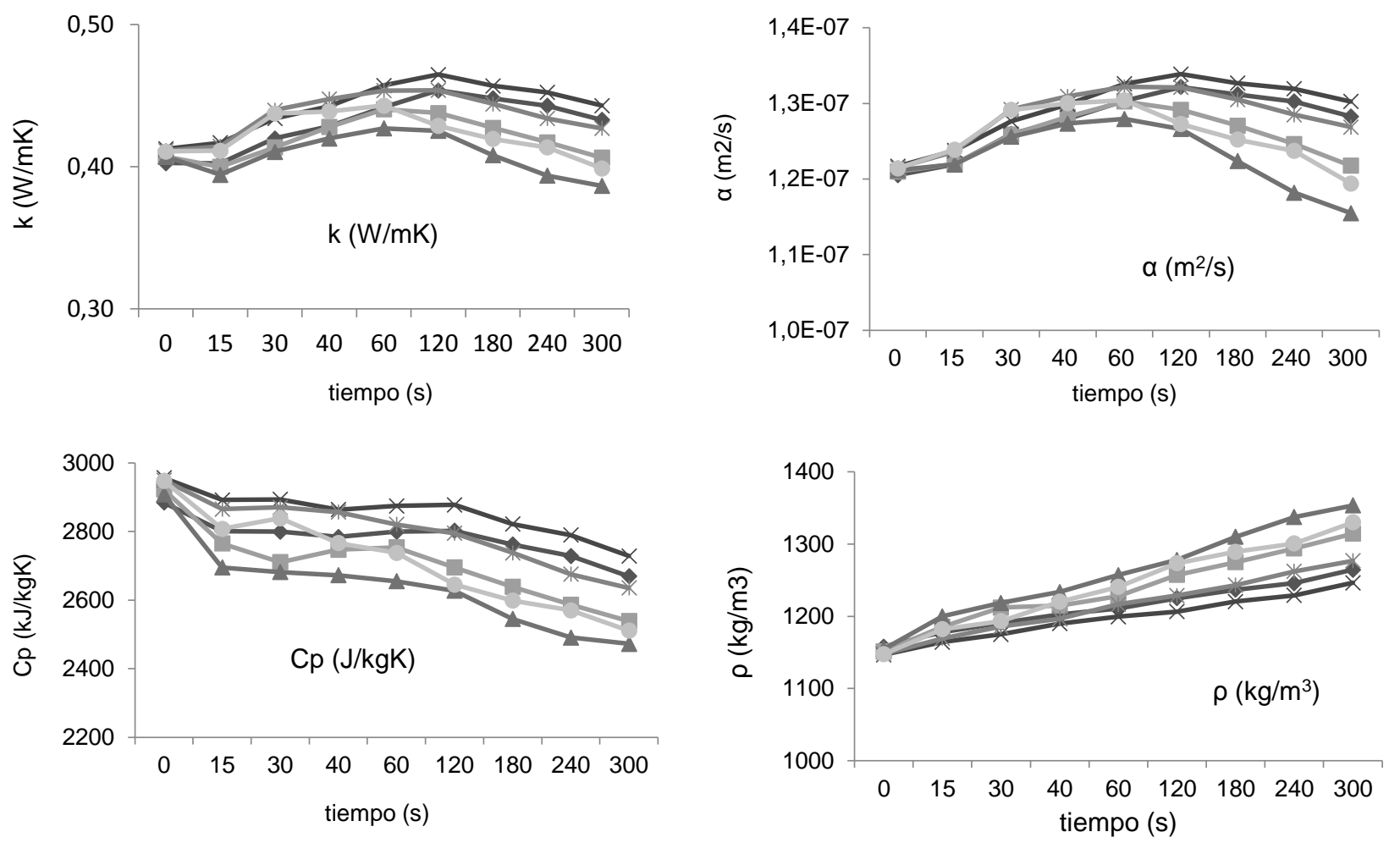

Fig. 1. Comportamiento de las propiedades termofísicas para diferentes tratamientos. SR sin recubrimiento y $\mathrm{CR}$ con recubrimiento $\rightarrow-150 \mathrm{SR} \rightarrow 170 \mathrm{SR} \rightarrow 190 \mathrm{SR} \approx-150 \mathrm{CR} * 170 \mathrm{CR} \rightarrow 190 \mathrm{CR}$

El mismo comportamiento lo tuvieron las muestras que fueron fritas a mayor temperatura; es decir, las muestras fritas a $190{ }^{\circ} \mathrm{C}$ tuvieron valores mayores de $\rho$ que las fritas a $170{ }^{\circ} \mathrm{C}$, y éstas a su vez que las de $150 \stackrel{\circ}{ } \mathrm{C}$. Esto se debe a la relación directa que existe entre estas propiedades y el contenido de humedad y temperatura que se describe en los modelos utilizados para calcularlos y ratifica que estas propiedades no pueden considerarse constantes durante el proceso de fritura. Durante el proceso de freído, y según lo observado en la figura 1, los valores de la conductividad y difusividad térmica aumentaron inicialmente mientras aumentó la temperatura, pero disminuyeron cuando la disminución del contenido de humedad fue representativo; esto se debe a que la formación de la costra empieza a atribuirle al alimento propiedades termofísicas similares a las de un material de aislamiento debido al aumento de su porosidad (Farinu y Baik, 2005; Farinu y Baik, 2007a; Alvis et al., 2009b). El calor específico, aunque también es influenciado por la temperatura del centro del alimento según las relaciones empíricas, su valor disminuye en el tiempo porque la relación con la humedad es más marcada (Alvis et al., 2009b). Todo esto permite que varios autores consideren las propiedades termofísicas constantes. Por ejemplo, Kim et al. (2011) consideraron valores constantes de $k$ igual a $0.554 \mathrm{~W} / \mathrm{mK}$ y $\alpha$ igual $1,45 \times 10^{-7} \mathrm{~m}^{2} / \mathrm{s}$ durante el estudio de la transferencia de calor en trozos de papa a $170^{\circ} \mathrm{C}$; Bouchon y Pyle (2005) en el estudio de la absorción de aceite durante el enfriamiento post-freído de papa, utilizaron valores constantes de las propiedades termofísicas, para la costra $\rho$ igual a $386 \mathrm{~kg} / \mathrm{m}^{3}, C p$ igual a $3050 \mathrm{~J} / \mathrm{kgK}$ y $\mathrm{k}$ igual a $0.14 \mathrm{~W} / \mathrm{mK}$; y para el centro del alimento $\rho$ igual a $1107 \mathrm{~kg} / \mathrm{m3}, C p$ igual a $3450 \mathrm{~J} / \mathrm{kgK}$ y $\mathrm{k}$ igual a $0.655 \mathrm{~W} / \mathrm{mK}$.

Con respecto a la densidad, se puede decir que esta propiedad aumenta a medida que disminuye la humedad. Aunque Farinu y Baik (2007a) consideraron que la densidad aumenta cuando disminuye la temperatura del alimento, en nuestro caso el aumento en la temperatura de freído ocasiona una mayor pérdida de humedad y por lo tanto valores más altos de densidad cuando la temperatura de freído es mayor. El uso de un recubrimiento comestible, si se comparan los tratamientos a la misma temperatura de freído, permite que las muestras tengan mayor valor de conductividad, difusividad y calor específico y menores valores de densidad. Esto se debe a que los recubrimientos comestibles, como el CMC utilizado, retienen la humedad del alimento y no permite que haya migración hacia el exterior de la muestra. De esta manera, el contenido de humedad de las muestras con recubrimiento es mayor que las muestras que no tienen recubrimiento. Los valores obtenidos en este estudio son similares a los obtenidos experimentalmente y reportados por varios autores. Farinu y Baik (2007a) obtuvieron experimentalmente para la batata, a una temperatura entre 20 y $60{ }^{\circ} \mathrm{C}$ y contenido de humedad entre 0.45 y 0.75 , valores de conductividad entre 0.452 a $0.528 \mathrm{~W} / \mathrm{mK}$, calor específico de 3182.6 a $4137.4 \mathrm{~J} / \mathrm{kgK}$, densidad entre 1138.5 
y $1285.5 \mathrm{~kg} / \mathrm{m}^{3}$ y difusividad térmica entre $1.11 \times 10^{-7}$ y $1.29 \times 10^{-7} \mathrm{~m}^{2} / \mathrm{s}$. Cabe resaltar que Choi y Okos (1985) ya habían demostrado que las propiedades termofísicas variaban con la temperatura y el contenido de humedad. Además, a pesar de proporcionar relaciones matemáticas para todos los tipos de alimentos, se obtienen valores similares a los obtenidos en este estudio.

Varios autores demostraron el mismo comportamiento de las propiedades termofísicas y consideraron que estas propiedades tienen dependencia del contenido de humedad, la temperatura y los demás componentes. Entre ellos tenemos a Sosa-Morales et al. (2006) durante el freído por inmersión de carne de cerdo, Taiwo y Baik (2007) en rodajas de batata fritas a $170 \stackrel{\circ}{\circ}$, Marcotte et al. (2008) en productos procesados de carne de res y de aves, Bon et al. (2010) en la pulpa de mango y Hassan y Ramaswamy (2011) en zanahorias y carne de res extra-magra. Adicionalmente, las diferencias entre los valores obtenidos y otros reportados se debe a que existe variación entre la composición de los diferentes tipos de alimentos, que pueden ser de diferentes variedades o incluso de la misma variedad pero que se producen bajo diferentes condiciones. Estas propiedades también dependen de muchos otros factores que afectan el flujo de calor en el alimento como el porcentaje de espacios vacíos y su disposición, la forma, el tamaño, la homogeneidad y la orientación de las fibras (Farinu y Baik 2007a).

\section{Propiedades de transporte}

\section{Coeficiente de transferencia de calor $h$.}

Los resultados de $h$ obtenidos en este estudio se muestran en la tabla 2 y tienen los siguientes valores promedios: para las muestras que no utilizaron recubrimiento el valor de $h$ fue de $144.22,168.15$ y 199.82 $W / m^{2} K$ para temperaturas de 150, 170 y $190 \stackrel{\circ}{ }$, respectivamente, y para las muestras con recubrimiento de CMC al $0.5 \%$ los valores de $h$ fueron $188.75,228.41$ y $239.06 \mathrm{~W} / \mathrm{m}^{2} \mathrm{~K}$ para las respectivas temperaturas.

Tabla 2. Coeficientes de transferencia de calor para los diferentes tratamientos. ${ }^{*}$ Diferencias estadísticas significativas

\begin{tabular}{|c|c|c|c|c|}
\hline & \multicolumn{2}{|c|}{ Sin Recubrimiento } & \multicolumn{2}{c|}{ Con Recubrimiento } \\
\hline Temperatura $\pm \mathrm{SD}\left({ }^{\circ} \mathrm{C}\right)$ & $\mathrm{Bi} \pm \mathrm{SD}$ & $h \pm \mathrm{SD}\left(W / m^{2}{ }^{\circ} \mathrm{C}\right)$ & $\mathrm{Bi} \pm \mathrm{SD}$ & $h \pm \mathrm{SD}\left(W / m^{2}{ }^{\circ} \mathrm{C}\right)$ \\
\hline 150 & $1.70 \pm 0.28$ & $144.22 \pm 25.61$ & $2.15 \pm 0.37$ & $188.75 \pm 36.03$ \\
\hline 170 & $2.00 \pm 0.18$ & $168.15 \pm 13.54^{\star}$ & $2.57 \pm 0.14$ & $228.41 \pm 11.73^{\star}$ \\
\hline 190 & $2.41^{*} \pm 0.21$ & $199.82 \pm 16.66^{*}$ & $2.73 \pm 0.15$ & $239.06 \pm 17.45^{\star}$ \\
\hline
\end{tabular}

En el análisis de varianza se observó un efecto altamente significativo para la temperatura y el recubrimiento, pero no se observó efecto de la interacción entre estos dos factores. Según la comparación de medias de Tukey, sólo hay diferencia significativa entre los valores promedios de $h$ obtenidos entre las temperaturas de 150 y $190{ }^{\circ} \mathrm{C}$. Los valores promedios para las muestras con recubrimiento fueron mayores que las que no lo utilizaron y a una temperatura de $190 \stackrel{\circ}{\circ}$ se obtuvieron los mayores valores de $h$.

A mayor temperatura mayor es el coeficiente convectivo de transferencia de calor debido al aumento en la diferencia de temperaturas entre la capa de aceite que permanece sobre la superficie y la temperatura superficial, lo que se convierte en un mayor flujo de calor (Sahin et al., 1999; Bouchon y Pyle, 2005; Butžaki y Seruga, 2005; Farinu y Baik, 2005; Sosa-Morales et al., 2006; Alvis et al., 2009a), también porque la viscosidad es menor y la agitación del burbujeo aumenta la turbulencia (Farinu y Baik, 2007b).

Con recubrimiento comestible los valores de $h$ fueron mayores. Matemáticamente, este comportamiento se puede explicar de la siguiente manera: siendo la conductividad mayor en el producto con recubrimiento, el flujo de calor por conducción se hace mayor; como en estado unidimensional, sin generación de calor, el calor por convección es igual al flujo de calor por conducción (Incropera y Dewitt, 1999). Para los mismos gradientes de temperatura entre el aceite y la superficie se hace mayor $h$ para cumplir con la igualdad. Los valores obtenidos en este estudio se encuentran dentro del rango reportado para productos vegetales entre 90 y $1100 \mathrm{~W} / \mathrm{m}^{2}{ }^{\circ} \mathrm{C}$ (Yildiz et al., 2007; Alvis et al., 2009a). Autores que utilizaron esta misma metodología para determinar el coeficiente de transferencia de calor (Alvis et al., 2009a; Kim et al., 2011) obtuvieron resultados similares. Alvis et al. (2009a) determinó el coeficiente $h$ para temperaturas de 140,160 y $180^{\circ} \mathrm{C}$ durante el freído de trozos de ñame, los respectivos resultados fueron $148.10,192.08$ y $204.46 \mathrm{~W} / \mathrm{m}^{2}{ }^{\circ} \mathrm{C}$.

Los resultados contrastan con los valores obtenidos por otros autores. Kim et al. (2011) determinaron los coeficientes convectivos de transferencia de calor en trozos de papa sin recubrimiento en $250.8 \mathrm{~W} / \mathrm{m}^{2} \mathrm{~K}$ y con recubrimiento $244.9,240.5$ y $230.0 \mathrm{~W} / \mathrm{m}^{2} \mathrm{~K}$ en concentraciones de $0.3,0.6$ y $0.9 \%$, respectivamente, para goma gellan y valores correspondientes de 242.2, 238.3 y $217.4 \mathrm{~W} / \mathrm{m}^{2} \mathrm{~K}$ para el recubrimiento con 
goma guar. Los motivos por los cuales con el recubrimiento se obtiene menor coeficiente de transferencia de calor se deben a que el recubrimiento no fue escurrido ni secado, proporcionando una resistencia adicional a la transferencia de calor. Además, como lo explica este mismo autor, a mayor concentración de goma más gruesa es la capa de recubrimiento y disminuye el valor de $h$. También, Yildiz et al (2007) determinaron que $h$ durante el freído de trozos de papa disminuían con el aumento de la temperatura, los valores promedios fueron $286.7,227.3$ y $181.3 \mathrm{~W} / \mathrm{m}^{2} \mathrm{~K}$ para las respectivas temperaturas de 150,170 y 190 ${ }^{\circ} \mathrm{C}$. Las razones de este comportamiento se basaron en que a mayor temperatura "se presenta mayor velocidad pérdida de humedad, y por lo tanto mayor es la cantidad extraída de energía entrante, lo que reduce la energía disponible para el aumento interior de la energía, lo que hace que disminuya $h$ ". Sin embargo, estos autores reconocen que sus resultados son contrarios a otros estudios reportados.

Utilizando otras metodologías para la determinación de $h$ varios autores han reportado resultados similares. Sahin et al, (1999) obtuvieron valores de $h$ de 90, 120, 150, 170 y $200 \mathrm{~W} / \mathrm{m}^{2} \mathrm{~K}$ durante el freído de masa de papa a 150, 160, 170, 180 y $190^{\circ} \mathrm{C}$, respectivamente. Vitrac et al. (2002) utilizaron un método indirecto para medir $h$ en una rodaja de aluminio y rociando finamente agua sobre la superficie del aceite a $160{ }^{\circ} \mathrm{C}$ para simular el burbujeo obtuvieron valores de $h$ de $160 \mathrm{~W} / \mathrm{m}^{2} \mathrm{~K}$ para el caso de convección natural sin agitación y alcanzó valores de 300 y $400 \mathrm{~W} / \mathrm{m}^{2} \mathrm{~K}$ cuando se presentó agitación de 150 y 300 rpm, respectivamente. También, reportó un rango de valores para $h$ entre 300 y $1300 \mathrm{~W} / \mathrm{m}^{2} K$ dependiendo del burbujeo presente. Sin embargo este método se considera que no estima bien $h$, especialmente durante el burbujeo, porque la pieza cambia inevitablemente la naturaleza y el patrón de flujo de las burbujas que proceden del alimento (Farinu y Baik, 2005; Butžaki y Šeruga (2005) determinaron $h$ durante el freído de esferas de papa de 2.5 $\mathrm{cm}$ de radio y los valores obtenidos al inicio del freído se encontraron entre 197.25 y $774.88 \mathrm{~W} / \mathrm{m}^{2} \mathrm{~K}$ para diferentes composiciones de la masa con harina de trigo y de papa; sin embargo, los mayores valores obtenidos fueron aproximadamente de 650, 700, 750 y $780 \mathrm{~W} / \mathrm{m}^{2} \mathrm{~K}$ para temperaturas de 160, 170, 180 y $190^{\circ} \mathrm{C}$, respectivamente; luego el $h$ convectivo disminuyó a medida que fue aumentando la corteza y se mantuvo relativamente constante alrededor de valores de $100 \mathrm{~W} / \mathrm{m}^{2} \mathrm{~K}$. Sosa-Morales et al. (2006) obtuvieron valores del coeficiente de transferencia de calor de $174.38,193.45$ y $226.10 \mathrm{~W} / \mathrm{m}^{2} \mathrm{~K}$ para unas temperaturas respectivas de 90,100 y $110^{\circ} \mathrm{C}$ durante el freído por inmersión de carne de cerdo.

Farinu y Baik (2007b) determinaron el valor de $h$ en el tiempo para rodajas de batata de diferentes diámetros. En los primeros segundos de fritura alcanzó valores máximos de 710, 722,760 y $837 \mathrm{~W} / \mathrm{m}^{2} \mathrm{~K}$ para las temperaturas de 150, 160, 170 y $180^{\circ} \mathrm{C}$ pero luego disminuyeron y se estabilizaron en un rango de 450 a $550 \mathrm{~W} / \mathrm{m}^{2} K$. Erdogdu y Dejmek (2010) determinaron el valor de $h$ para diferentes tiempos de freído de rodajas gruesas de papa a $170^{\circ} \mathrm{C}$ y obtuvieron valores de $265 \mathrm{~W} / \mathrm{m}^{2} \mathrm{~K}$ para una presión atmosférica y de $500 \mathrm{~W} / \mathrm{m}^{2} \mathrm{~K}$ para una presión de $1.97 \mathrm{~atm}$. Varios autores, en lugar de determinar $h$ experimentalmente, han utilizado valores de referencia para el coeficiente convectivo durante estudios y simulaciones relacionadas con la transferencia de calor durante el freído. Bouchon y Pyle (2005) utilizaron valores para el coeficiente convectivo tomados de datos conocidos a diferentes temperaturas para estudiar la modelación de la absorción del aceite durante el enfriamiento post-fritura. Los valores que utilizaron fueron constantes para una rodaja infinita de papa frita a $180^{\circ} \mathrm{C}$ de $277 \mathrm{~W} / \mathrm{m}^{2} \mathrm{~K}$ durante convección natural y $500 \mathrm{~W} / \mathrm{m}^{2} \mathrm{~K}$ durante la etapa de burbujeo y para un cilindro infinito utilizó unas correlaciones que indican la variación de $h$ con el tiempo de fritura. González-Martínez et al. (2004) utilizaron el valor de $750 \mathrm{~W} / \mathrm{m}^{2} \mathrm{~K}$ en la simulación de la penetración de calor durante el escaldado de papas en forma de trozo de $2 \times 7.5 \times 2.5 \mathrm{~cm}^{3}$. Sin embargo, existen un rango teórico donde se encuentran los valores para el coeficiente $h$ entre 250 y $1100 \mathrm{~W} / \mathrm{m}^{2} \mathrm{~K}$ (Farinu y Baik, 2005) y estas diferencias se pueden justificar debido a las características de los productos, los métodos utilizados para medir la temperatura del alimento y el método de solución de las ecuaciones de transferencia de calor (Alvis et al., 2009a).

\section{Coeficiente de transferencia de masa kc.}

Para calcular el coeficiente de transferencia de masa no se tuvo en cuenta el comportamiento de la velocidad de evaporación durante los primeros 15 segundos. Dicha fase se caracteriza por la evaporación repentina de la humedad superficial y puede no ser lineal porque hay inestabilidad debido a la turbulencia en el aceite cerca de la superficie causado por el burbujeo del vapor de agua (Kassama y Ngadi, 2005; Yildiz et al., 2007). Sin embargo, luego de esos primeros segundos se considera una etapa de velocidad de evaporación constante hasta que se presenta la etapa de velocidad de evaporación decreciente y se desarrolla la costra gracias a que el frente de evaporación se desplaza hacia el interior del producto (Bouchon y Pyle, 2005).

Los valores promedios obtenidos para $k c$ en este estudio se muestran en la tabla 3 , a las respectivas temperaturas de 150,170 y $190^{\circ} \mathrm{C}$, son de $3.81 \times 10^{-4}, 7.66 \times 10^{-4}$ y $10.24 \times 10^{-4} \mathrm{~m} / \mathrm{s}$ para las muestras sin recubrimiento y $3.57 \times 10^{-4}, 4.78 \times 10^{-4}$ y $7.67 \times 10^{-4} \mathrm{~m} / \mathrm{s}$ para las muestras con recubrimiento. Por su parte, los valores promedios obtenidos para $D$ en este estudio, a las respectivas temperaturas de 150,170 y $190^{\circ} \mathrm{C}$, 
son de $9.19 \times 10^{-7}, 10.7 \times 10^{-7}$ y $13.9 \times 10^{-7} \mathrm{~m}^{2} / \mathrm{s}$ para las muestras sin recubrimiento y $9.52 \times 10^{-7}, 12.3 \times 10^{-7}$ y $15.3 \times 10^{-7} \mathrm{~m}^{2} / \mathrm{s}$ para las muestras con recubrimiento. En el análisis de varianza se observó un efecto altamente significativo para la temperatura y el recubrimiento, pero no se observó efecto de la interacción entre estos dos factores. Según la comparación de medias de Tukey, sólo no hay diferencia significativa entre los valores promedios de $k c$ obtenidos a las temperaturas de 150 y $170 \stackrel{\circ}{\circ}$. Los valores promedios de $k c$ para las muestras sin recubrimiento fueron mayores que para las muestras con recubrimiento. Además, a una temperatura de $190 \stackrel{\circ}{ } \mathrm{C}$ se obtuvieron los mayores valores de $k c$. Los valores experimentales de $k c$ y $D$ son mayores para las temperaturas más altas, así como lo ratifican otros autores (Farinu y Baik, 2005; Yildiz et al., 2007; Farinu y Baik, 2008; Alvis et al., 2009a; Moreira et al., 2009; Mosavian y Karizaki, 2012), quienes afirman que la velocidad de pérdida de humedad tiende a ser mayor a mayor temperatura del aceite, lo mismo que la difusividad efectiva debido a que a mayor temperatura mayor porosidad y consecuentemente mayor difusividad del agua. De igual manera, afirman que los cambios físicos que se presentan durante la fritura, como el encogimiento, conducen a mayores errores en el cálculo de la difusividad (Kassama y Ngadi, 2005; Mai Tran et al., 2007).

Tabla 3. Coeficientes de transferencia de masa $k c$ para los diferentes tratamientos.

\begin{tabular}{|c|c|c|c|}
\hline & \multicolumn{3}{|c|}{ Sin Recubrimiento } \\
\hline $\begin{array}{c}\text { Temperatura } \\
\left({ }^{\circ} \mathrm{C}\right)\end{array}$ & $\begin{array}{c}D \pm \mathrm{SD} \\
\times 10^{6}\left(\mathrm{~m}^{2} / \mathrm{s}\right)\end{array}$ & Bim $\pm \mathrm{SD}$ & $\begin{array}{c}k c \pm \mathrm{SD} \\
\times 10^{4}(\mathrm{~m} / \mathrm{s})\end{array}$ \\
\hline 150 & $0.92 \pm 0.17$ & $2.12 \pm 0.37$ & $3.81 \pm 0.12$ \\
\hline 170 & $1.07 \pm 0.14$ & $3.67 \pm 0.89$ & $7.66 \pm 0.76$ \\
\hline 190 & $1.39 \pm 0.38$ & $4.05 \pm 2.05$ & $10.2 \pm 2.62$ \\
\hline & \multicolumn{3}{|c|}{ Con Recubrimiento } \\
\hline $\begin{array}{c}\text { Temperatura } \\
\left({ }^{\circ} \mathrm{C}\right)\end{array}$ & $\begin{array}{c}\mathrm{D} \pm \mathrm{SD} \\
\mathrm{x} 10^{6}\left(\mathrm{~m}^{2} / \mathrm{s}\right)\end{array}$ & Bim $\pm \mathrm{SD}$ & $k \mathrm{SD}$ \\
\hline 150 & $0.95 \pm 0.22$ & $1.97 \pm 0.57$ & $3.57 \pm 0.34$ \\
\hline 170 & $1.23 \pm 0.12$ & $1.96 \pm 0.37$ & $4.78 \pm 0.42$ \\
\hline 190 & $1.53 \pm 0.36$ & $2.70 \pm 1.19$ & $7.67 \pm 1.27$ \\
\hline
\end{tabular}

Mai Tran et al. (2007) y Durán et al. (2007) justificaron los errores existentes en el papel que cumple la costra al impedir el movimiento de las burbujas de vapor; en este caso, el vapor de agua ejerce una presión sobre la costra y la revienta, ocasionando una pérdida de humedad aleatoria y poco predecible, lo que conlleva a diferencias en los resultados obtenidos en cada muestra. En nuestro caso, los errores mayores se presentaron a mayores temperaturas, donde los cambios físicos se observan más rápidamente. El recubrimiento hace que disminuya el coeficiente de transferencia de masa. Esto se debe a que durante la difusión del agua a través del centro del alimento y de la costra encuentra una resistencia adicional que corresponde a la película de recubrimiento que se encuentra en la superficie y que el alimento tratado no la tiene (Varela y Fiszman, 2011), también $k c$ es una función del gradiente de humedad en el producto y estos gradientes se hacen menores gracias a la poca remoción que se experimenta cuando el recubrimiento es aplicado (Yamsaengsung y Moreira, 2002). Es importante aclarar que las condiciones durante la formación de la película influencian en sus características finales como la resistencia, la flexibilidad, la permeabilidad y la uniformidad en el espesor de la barrera. Si el recubrimiento no tiene grietas y se encuentra uniforme, el vapor fluye a través de la película por difusión, se solubiliza en la matriz del recubrimiento gracias al gradiente de concentración de agua que existe con la superficie externa de la película (Varela y Fiszman, 2011).

Los cambios en la estructura del alimento, especialmente en la estructura de los poros, afectan la difusividad de los gases y de los líquidos en el material (Yamsaengsung y Moreira, 2002). Cuando existe saturación de agua en las diferentes regiones del alimento, la difusión por capilaridad es relativamente constante (Farinu y Baik, 2005). Los resultados del coeficiente de difusión son mayores a los reportados en diferentes estudios y del rango reportado por Alvis et al. (2009a) entre $1.31 \times 10^{-7}$ y $24 \times 10^{-10} \mathrm{~m}^{2} / \mathrm{s}$ y mucho mayores a los reportados por Farinu y Baik (2005) entre $58 \times 10^{-10}$ y $109 \times 10^{-10} \mathrm{~m}^{2} / \mathrm{s}$ durante fritura para diferentes productos alimenticios, aclarando que las diferencias pueden ser atribuidas a la naturaleza del producto, las condiciones del proceso como la temperatura y los métodos de determinación (Alvis et al., 2009a). Sin embargo, se encuentran más cerca a los valores obtenidos experimentalmente por Yildiz et al. (2007) de $1.12 \times 10^{-5}, 1.58 \times 10^{-5}, 2.07 \times 10^{-5} \mathrm{~m} / \mathrm{s}$ para las respectivas temperaturas de 150,170 y $190{ }^{\circ} \mathrm{C}$. Los valores de difusividad fueron, para las mismas temperaturas, de $9.2 \times 10^{-9}, 11.0 \times 10^{-9}$ y $18.2 \times 10^{-9} \mathrm{~m}^{2} / \mathrm{s}$. 
Otros resultados obtenidos por otros autores se muestran a continuación. Costa y Oliveira (1999) estimaron la difusividad para la pérdida de humedad en trozos de papa tipo francés a $140 \stackrel{\circ}{\mathrm{C}}$ de $4,92 \times 10^{-9} \mathrm{~m}^{2} / \mathrm{s}$ y a 180 ${ }^{\circ} \mathrm{C}$ de $6,93 \times 10^{-9} \mathrm{~m}^{2} / \mathrm{s}$. Kassama y Ngadi (2005) obtuvieron valores de difusividad efectiva en carne de pollo entre $3,65 \times 10^{-9}$ y $7,42 \times 10^{-9} \mathrm{~m}^{2} / \mathrm{s}$ en rangos de temperatura de 170 a $190{ }^{\circ} \mathrm{C}$ durante 900 segundos de freído. Sosa-Morales et al. (2006) obtuvieron valores del difusividad entre 1.59 y $30.2 \times 10^{-9} \mathrm{~m}^{2 / \mathrm{s}}$ para unas temperaturas entre 90 y $110^{\circ} \mathrm{C}$ durante el freído por inmersión de carne de cerdo. Farinu y Baik (2008) estimaron el coeficiente $k c$ mediante simulación del proceso de freído de rodajas de batata y obtuvieron valores máximos de $4.9 \times 10^{-6}, 5.7 \times 10-6,7.0 \times 10^{-6}$ y $7.3 \times 10^{-6} \mathrm{~m} / \mathrm{s}$ para temperaturas de freído de 150, 160, 170 y $180^{\circ} \mathrm{C}$, respectivamente. Alvis et al. (2009a) para el coeficiente kc indican valores de $0.78 \times 10^{-6}$, $1.77 \times 10^{-6}, 3.30 \times 10^{-6} \mathrm{~m} / \mathrm{s}$ para temperaturas de 140,160 y $180^{\circ} \mathrm{C}$, respectivamente, y valores de difusividad de $1.95 \times 10^{-9}, 2.59 \times 10^{-9}$ y $3.24 \times 10^{-9} \mathrm{~m}^{2} / \mathrm{s}$ durante el freído de trozos de ñame. Mosavian y Karizaki (2012) determinaron la difusividad efectiva y el coeficiente de transferencia de masa en galletas de arroz con y sin harina de pescado a diferentes temperaturas. Los resultados de $k c$ fueron para las galletas con harina de pescado de $5.51 \times 10^{-6}, 7.06 \times 10^{-6}$ y $9.31 \times 10^{-6} \mathrm{~m} / \mathrm{s}$ y paras las que no tenían harina de pescado de $5.98 \times 10^{-6}$, $7.46 \times 10^{-6}$ y $9.70 \times 10^{-6} \mathrm{~m} / \mathrm{s}$ para 150,170 y $190^{\circ} \mathrm{C}$. Para las mismas temperaturas, los coeficientes de difusividad $D$ efectiva fueron $1.24 \times 10^{-8}, 1.60 \times 10^{-8}$ y $2.36 \times 10^{-8} \mathrm{~m}^{2} / \mathrm{s}$ con harina de pescado y $1.27 \times 10^{-8}$, $1.61 \times 10^{-8}$ y $2.36 \times 10^{-8} \mathrm{~m}^{2} / \mathrm{s}$ sin harina de pescado.

\section{Pérdida de humedad y absorción de aceite}

Los porcentajes de humedad y de grasa se muestran en la figura 2. El resultado del análisis de varianza para la pérdida de humedad y la absorción de aceite indicó que hay un efecto altamente significativo del factor de bloqueo, de la temperatura y del recubrimiento, pero no se encontró efecto de la interacción de los factores temperatura y recubrimiento. Mediante una prueba de Tukey se encontraron que todas las diferencias en el contenido de humedad fueron significativas, teniendo para las muestras con recubrimiento un contenido de humedad mayor que para las muestras sin recubrimiento. El mismo comportamiento lo tuvieron las muestras que fueron fritas a menor temperatura; es decir, las muestras fritas a $150 \stackrel{\circ}{C}$ tuvieron

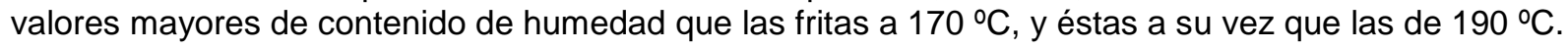
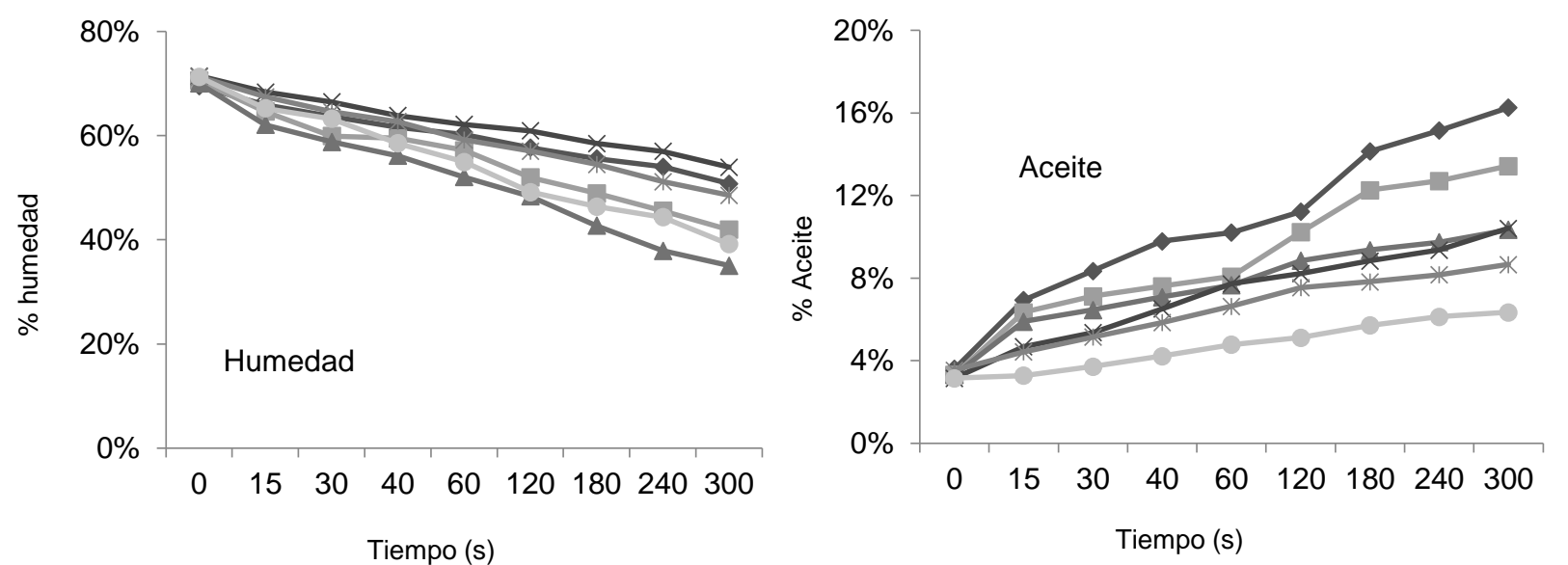

Fig. 2. Comportamiento del contenido de humedad y de aceite durante el freído.

$$
\multimap 150 \mathrm{SR} \longrightarrow-170 \mathrm{SR} \longleftarrow 190 \mathrm{SR} \approx 150 \mathrm{CR} \approx-170 \mathrm{CR} \longrightarrow 190 \mathrm{CR}
$$

En el caso de la absorción del aceite, mediante una prueba de Tukey se encontraron que todas las diferencias en el contenido de aceite absorbido fueron significativas, teniendo que para las muestras sin recubrimiento el contenido de aceite fue mayor que para las muestras sin recubrimiento. El mismo comportamiento lo tuvieron las muestras que fueron freídas a menor temperatura; es decir, las muestras freídas a $150{ }^{\circ} \mathrm{C}$ tuvieron valores mayores en el contenido de aceite que las freídas a $170 \stackrel{\circ}{\circ}$, y éstas a su vez que las de $190^{\circ} \mathrm{C}$.

Durante el proceso de fritura por inmersión se crean poros en el producto ocasionados por la pérdida de humedad, generando un espacio destinado para la entrada de aceite. Cuando hay un alto contenido de agua, ésta fluye en el medio poroso debido a las fuerzas capilares hasta el frente de evaporación (Kassama y Ngadi, 2005). Por esta razón, la formación de estos capilares controla la absorción del aceite. Sin embargo, también se ha demostrado que durante la pérdida de humedad se presenta un encogimiento en las dimensiones del producto (Kassama y Ngadi, 2005), lo que hace que los poros disminuyan su tamaño. 


\section{Efecto de la temperatura}

A mayor temperatura de freído se observó mayor pérdida de humedad y menor absorción de aceite como lo ratifican otros estudios (Rimac-Brnčić et al., 2004; Moyano y Pedreschi, 2006; Durán et al., 2007; Pedreschi et al., 2008; Marcano et al., 2010; Alvis et al., 2010). Todos estos coinciden en afirmar que la etapa de absorción de aceite es un fenómeno complejo que ocurre principalmente cuando el producto es removido del aceite, durante la etapa de enfriamiento (Bouchon y Pyle, 2005; Durán et al., 2007; Farinu y Baik, 2008; Pedreschi et al., 2008; Ziaiifar et al., 2008; Moreira et al., 2009; Alvis et al., 2010).

Lo anterior se debe a diferentes factores: cuando se frita a mayor temperatura aumenta la velocidad de evaporación del agua (Atehortúa et al., 2005; Farinu y Baik, 2007b; Yildiz et al., 2007; Alvis et al., 2009a) y por lo tanto es más rápida la pérdida de humedad que genera huecos capilares y vacíos en la estructura del alimento. Esta porosidad juega un papel muy importante en la absorción (Yamsaengsung y Moreira, 2002) ya que el aceite se adhiere a la superficie mientras las presiones internas mayores impiden la penetración en la estructura del producto (Pedreschi et al., 2008) y se absorbe en los poros sólo cuando el producto sale del aceite y se enfría (Mai Tran et al., 2007) y el manto de vapor alrededor del alimento frito desaparece. En este momento se desarrolla extremadamente rápido un gradiente positivo de presión que produce un efecto de vacío que genera la fuerza que succiona el aceite dentro de los poros, a mayor coeficiente convectivo en el medio donde se enfría el producto más rápida será la absorción de aceite (Yamsaengsung y Moreira, 2002; Bouchon y Pyle, 2005; Durán et al., 2007).

Hay que tener en cuenta que, además del encogimiento que se presenta, a mayor temperatura también se gelatinizan más rápido los almidones y el porcentaje de agua libre se agota en el producto creando una barrera para el escape de vapor que causa una expansión abrupta en los poros de capilaridad, que al aumentar su tamaño disminuyen la presión capilar y la absorción de aceite (Durán et al., 2007; Taiwo y Baik, 2007; Ziaiifar et al., 2008; Moreira et al., 2009). Otro factor importante se presenta cuando la mayoría del agua se ha evaporado y la temperatura del producto aumenta, entonces la tensión superficial disminuye y la velocidad de absorción de aceite disminuye (Durán et al., 2007; Ziaiifar et al., 2008), aunque aumente el área de contacto con el producto (Kim et al., 2011) porque el aceite puede eliminarse fácilmente mediante el drenaje, contribuyendo a disminuir la diferencia de presiones en los poros del producto (Ziaiifar et al., 2008).

Además, a mayor temperatura disminuyen los tiempos de freído, factor que afecta directamente la absorción de aceite (Rimac-Brnčić et al., 2004; Kita et al., 2007; Marcano et al., 2010) y que, según Atehortúa et al. (2005) tiene más influencia en el contenido de aceite absorbido que la temperatura. Otras variables que afectan la absorción de aceite son las características fisicoquímicas del producto como la composición, la forma, la relación área superficial - peso y la porosidad (Rimac-Brnčić et al., 2004). Bouchon y Pyle (2005) estudiaron la absorción de aceite durante el tiempo de enfriamiento y encontraron que el tiempo necesario para permitir la absorción de aceite depende principalmente del grosor de la capa de la corteza, la temperatura ambiente, el coeficiente transferencia de calor por convección entre el aire y la superficie del producto y el radio de los poros.

Durán et al. (2007) estudiando la cinética de absorción de aceite definieron tres fracciones de aceite en el producto: el estructural que es el absorbido durante la fritura, el que penetra en la superficie durante el enfriamiento y el que se mantiene en la superficie y no penetra. Por esta razón indicaron que la superficie del producto frito juega un papel muy importante para la retención del aceite y la tendencia ha mostrado el uso de recubrimientos que permitan modificar dicha superficie y disminuya el contenido de aceite. Sin embargo, Kassama y Ngadi (2005) encontraron que a mayor temperatura de freído se alcanza mayor absorción del aceite. Esto fue justificado porque consideran la pérdida de humedad y la absorción de aceite como procesos simultáneos durante el freído, ya que a mayor temperatura se alcanza mayor porosidad donde el aceite llena los vacíos producidos por la eliminación del agua.

Durán et al. (2007) durante estudios con fritura de papas en rodajas, obtuvieron que el $38 \%$ del aceite es absorbido durante la fritura y casi el $62 \%$ permanece en la superficie sin penetrar. Ya durante el enfriamiento el $65 \%$ es absorbido y el $35 \%$ permanece en la superficie. Esta última relación fue obtenida por Pedreschi et al. (2008) pero sus resultados indicaron una absorción del $75 \%$ del aceite en los primeros cuatro minutos de fritura. Moreira et al. (2009) demostraron que durante los primeros 120 segundos se alcanza mayor absorción de aceite a mayor temperatura de freído porque se encuentra mayor cantidad de espacios vacíos, pero con el transcurso del tiempo esta humedad se va eliminando paulatinamente hasta dejar libre el espacio que luego es ocupado por el aceite y obtener mayor absorción de aceite a menor temperatura. Además, estos autores consideran que la fritura a presión atmosférica permite que el aceite entre en el producto antes de ser eliminado de la superficie. 
Modelar la absorción de aceite es muy difícil, los autores no se ponen de acuerdo en cuál es la fuerza más importante; unos indican que es la capilaridad y otros la tensión superficial. En realidad, los factores más importantes en la absorción de aceite son: las propiedades del producto como el tamaño, la forma, la relación superficie/volumen y la composición; las propiedades del aceite de fritura como la viscosidad, la tensión superficial y el envejecimiento; los factores del proceso como los pretratamientos, el tiempo y la temperatura de fritura; y condiciones de post-fritura (Ziaiifar et al., 2008; Freitas et al., 2009) ya que se considera que la absorción de aceite se presenta como un balance entre las fuerzas de adhesión como la capilaridad y el drenaje o escurrido (Ziaiifar et al., 2008).

\section{Efecto del recubrimiento}

Aunque teóricamente se ha expresado que el contenido de aceite en un producto frito se encuentra sobre el 40\% del producto final (Rimac-Brnčić et al., 2004; Kita et al., 2007; Mai Tran et al., 2007; Freitas et al., 2009) donde la mayoría del aceite queda en la costra porque el centro constituye alimento cocido (Farinu y Baik, 2005), la tendencia de los consumidores se orienta a los productos sanos con menor cantidad de aceite ya que, nutricionalmente, el consumo de grasa es considerado el principal factor de sobrepeso y de enfermedades coronarias en la población (Priya et al., 1996; Durán et al., 2007; Usawakesmanee et al., 2008; Ziaiifar et al., 2008; Varela y Fiszman, 2011), teniendo en cuenta que con la absorción de aceite también se absorben compuestos de alteración, incluso sustancias tóxicas con potencial cancerígeno como los compuestos polares (Marcano et al., 2010).

Las muestras tratadas con recubrimiento de carboximetil celulosa CMC absorbieron menor cantidad de aceite. El porcentaje de reducción obtenido fue de $35.93 \%, 36.22 \%$ y $42.14 \%$ para las temperaturas de 150,170 y $190 \stackrel{\circ}{\circ}$, respectivamente tomando como tiempo de freído el que proporcionó una humedad promedio de $49.32 \% \pm 1,27 \%$ así: 300,180 y 120 segundos para temperaturas respectivas de 150,170 y $190^{\circ} \mathrm{C}$. Esto se debe a que la función más importante que cumple el CMC es la de evitar la pérdida de humedad ya que tiene la capacidad de ligar el agua, y, por lo tanto, disminuye la cantidad de aceite que la puede sustituir (Priya et al., 1996; Rimac-Brnčić et al., 2004; Varela y Fiszman, 2011). Además, como es un polímero hidrofílico, forma una capa fina sobre la superficie del alimento que se comporta como barrera a la incorporación de aceite (Freitas et al., 2009; Varela y Fiszman, 2011); sin embargo, el grado de reducción depende de diferentes factores incluyendo el tipo de matriz alimenticia (Kim et al., 2011). Además, el CMC aumenta la tensión superficial, facilitando con mayor razón el escurrido del aceite superficial (Priya et al., 1996; Farinu y Baik, 2005; Ali et al., 2012).

La reducción en el contenido de aceite puede compararse con otros estudios donde se han obtenido resultados similares: Priya et al. (1996) lograron disminuir el contenido de aceite en productos fritos. Adicionando $2 \%$ CMC a las mezcla del producto, el contenido de aceite se redujo en $26.2 \%$ y adicionando $1 \%$ HPMC la reducción fue del $22.7 \%$. Usawakesmanee et al. (2008) incorporaron recubrimientos de HPMC, MC y Gluten de trigo en la mezcla que compone el apanado de papas junto con harina, azúcar, sal, especias y agua; y también obtuvieron resultados similares: con el uso de MC los resultados fueron más efectivos porque obtuvieron un contenido aceite final entre 22 y $24 \%$ aproximadamente frente a los valores obtenidos de $27 \%$ en la muestra control. La absorción de aceite con HPMC también fue significativa pero con valores de $25 \%$ aproximadamente mientras que el gluten de trigo no mostró reducción significativa en la reducción de aceite.

Rimac-Brnčić et al. (2004) estudiaron el efecto de diferentes tratamientos para evitar la absorción de aceite en trozos de papa fritos. El mejor resultado, con reducción del 54\%, lo obtuvieron al aplicar recubrimiento de solución de carboximetilcelulosa CMC al $1 \%$ en muestras que fueron escaldadas previamente con cloruro de calcio $\mathrm{CaCl}_{2}$. Varela y Fiszman (2011) utilizaron sorbitol como componente del recubrimiento de MC para mejorar las características de flexibilidad de la película y evitar las fisuras. Lograron reducir la cantidad de aceite absorbido en un $40.6 \%$ utilizando una solución de $1 \%$ MC y $0.5 \%$ sorbitol en tiras de papa. Estos resultados contrastan con los obtenidos por Durán et al. (2007), que indicaron que las muestras con recubrimiento de hidroxipropilmetilcelulosa HPMC absorbieron más aceite que las escaldadas en agua y las escaldadas en solución salina debido a la limitada capacidad del HPMC de formar recubrimientos gracias a la presencia de los grupos hidroxipropil.

Otros pretratamientos también han sido utilizados por diferentes investigadores para disminuir la absorción de aceite. Mai Tran et al. (2007) obtuvieron una reducción del $30 \%$ en la cantidad del aceite absorbido durante el freído de chips de papa a $180^{\circ} \mathrm{C}$ luego de escaldar las muestras y de sumergirlas en una solución de sacarosa al $23.1 \%$ peso. Da Silva y Moreira (2008) lograron reducir en un $24 \%$ el contenido de aceite en chips de batata y en un $16 \%$ en habichuelas verdes mediante fritura al vacío; Moreira et al. (2009) lograron reducir alrededor de un $85 \%$ el contenido de aceite en rodajas de papa fritas a $120 \stackrel{\circ}{\circ}$ en vacío. 
Freitas et al. (2009) obtuvieron mejores resultados en la aplicación de hidrocoloide a base de proteína de suero comparada con pectina y proteína aislada de soja. Kim et al. (2011) obtuvieron una reducción del $41 \%$ en trozos de papa fritos y recubiertos con solución de goma guar en una concentración del $0.9 \%$.

\section{CONCLUSIONES}

Las propiedades termofísicas de la batata varían durante la etapa de freído para la $k S R$ presentando valores de 0.39 a $0.45 \mathrm{~W} / \mathrm{mK}$ y $k C R$ valores entre 0.41 y $0.46 \mathrm{~W} / \mathrm{mK}$, el $C p$ SR valores entre 2470 a 2920 $\mathrm{kJ} / \mathrm{kg} \mathrm{K}$ y $C p C R$ valores entre 2500 a $2960 \mathrm{~kJ} / \mathrm{kg} \mathrm{K}$. la a $S R$ valores entre 1.16 a $1.35 \times 10^{-7} \mathrm{~m}^{2} / \mathrm{s}$ y $\alpha C R$ valores entre 1.19 a $1.34 \times 10^{-7} \mathrm{~m}^{2} / \mathrm{s}$ y para la $\rho$ la variación es más notoria con valores $\rho S R$ entre 1150 a $2920 \mathrm{~kg} / \mathrm{m}^{3}$ y $\rho C R$ entre 1150 a $2960 \mathrm{~kg} / \mathrm{m}^{3}$ por lo tanto se considera un error asumirlas constantes. A pesar de esto, cuando se estudian etapas muy cortas del freído, en nuestro caso la etapa de velocidad de pérdida de agua constante, se pueden utilizar los valores determinados para cada rango de tiempo.

El coeficiente de transferencia de calor $h$ es mayor cuando aumenta la temperatura del aceite y se utiliza recubrimiento comestible. Los valores obtenidos fueron de $144.22,168.15$ y $199.82 \mathrm{~W} / \mathrm{m}^{2}{ }^{\circ} \mathrm{C}$ para las muestras sin recubrimiento a temperaturas de 150, 170 y $190 \stackrel{\circ}{\circ}$, respectivamente, y de $188.75,228.41$ y $239.06 \mathrm{~W} / \mathrm{m}^{2}{ }^{\circ} \mathrm{C}$ para las mismas temperaturas. El coeficiente de transferencia de masa $\mathrm{kc}$ es mayor cuando aumenta la temperatura del aceite y no se utiliza recubrimiento comestible. Los valores obtenidos fueron de $3.81 \times 10^{-4}, 7.66 \times 10^{-4}, 10.20 \times 10^{-4} \mathrm{~m} / \mathrm{s}$ para las muestras sin recubrimiento a temperaturas de 150,170 y 190 ${ }^{\circ} \mathrm{C}$, respectivamente, y de $3.57 \times 10^{-4}, 4.78 \times 10^{-4}, 7.67 \times 10^{-4} \mathrm{~m} / \mathrm{s}$ para las mismas temperaturas.

El recubrimiento comestible disminuye más del $30 \%$ el contenido de aceite final para todas las temperaturas de freído utilizadas, lo que hace que sea una excelente elección para mejorar la calidad nutricional de los productos fritos.

\section{AGRADECIMIENTOS}

Los autores agradecen el apoyo logístico y financiero prestado por la Universidad de Córdoba, a través de la oficina de Investigación y extensión.

\section{REFERENCIAS}

Ali, H.S., A. G. Abdel-Razek, M. M. Kamil, Effect of pre-frying treatments of french fried potatoes to achieve better oil uptake reduction for health and technological aspects. Journal of Applied Sciences Research, 8 (10): 5018-5024 (2012).

Alvis, A., L. Cortés y M. Páez, Transferencia de calor y materia durante la fritura de trozos de ñame (Dioscórea alata). Información Tecnológica 20(1): 99-109 (2009a).

Alvis, A., C. Vélez, M. Rada-Mendoza, M. Villamiel y H. Villada, Heat transfer coefficient during deep-fat frying. Food Control 20: 321-325 (2009b).

Alvis, A., C. Vélez y G. Arrazola, Efecto de las condiciones de freído sobre la perdida de humedad y ganancia de aceite en trozos de ñame (Dioscórea alata). Ingeniería e Investigación 30(1): 41-44 (2010).

AOAC, Métodos de análisis de la asociación oficial de química analítica para determinar humedad, fibra, cenizas, grasa y proteína. Chapter 32: 1, 2, 5 y 14, Washington, U.S.A. (2003).

Atehortúa, A., C. Molina y A. Ortíz, Procesos de deshidratación impregnación por inmersión y prefritura aplicados a la elaboración de tajadas de plátano (Musa Paradisíaca AAB). Ingeniería y Competitividad 7(1): 56-64 (2005).

Bon, J., H. Váquiro, J. Benedito y J. Telis-Romero, Thermophysical properties of mango pulp (Mangifera indica L. cv. Tommy Atkins). Journal of Food Engineering 97: 563-568 (2010).

Bouchon, P. y D. Pyle, Modelling oil absorption during post-frying cooling II: Solution of the mathematical model, model testing and simulations. Food and Bioproducts Processing 83(C4): 261-272 (2005).

Budžaki, S. y B. Šeruga, Determination of convective heat transfer coefficient during frying of potato dough. Journal of Food Engineering 66: 307-314 (2005). 
Choi, Y. y M. Okos, Effects of temperature and composition on the thermal properties of foods. Food Engineering and Process Applications 1: 93-101 (1985).

Costa, R. y F. Oliveira, Modelling the kinetics of water loss during potato frying with a compartmental dynamic model. Journal of Food Engineering 41:177-185 (1999).

Da Silva, P. y R. Moreira, Vacuum frying of high-quality fruit and vegetable-based snacks. LWT - Food Science and Technology 41: 1758-1767 (2008).

Durán, M., F. Pedreschi, P. Moyano y E. Troncoso, Oil partition in pre-treated potato slices during frying and cooling. Journal of Food Engineering 81:257:265 (2007).

Erdogdu, F. y P. Dejmek, Determination of heat transfer coefficient during high pressure frying of potatoes. Journal of Food Engineering 96: 528-532 (2010).

Farinu, A. y O. Baik, Deep fat frying of foods-Transport Phenomena. Food Reviews International 21: 389410 (2005).

Farinu, A. y O. Baik, Thermal properties of sweet potato with its moisture content and temperature. International Journal of Food Properties 10: 703-719 (2007a).

Farinu, A. y O. Baik, Heat transfer coefficients during deep fat frying of sweetpotato: effects of product size and oil temperature. Food Research International 40: 989-994 (2007b).

Farinu, A. y O. Baik, Convective mass transfer coefficients in finite element simulations of deep fat frying of sweetpotato. Journal of Food Engineering 89: 187-194 (2008).

Farkas, B., R. Singh y T. Rumsey, Modeling heat and mass transfer in immersion frying. I Model development. Journal of Food Engineering 29: 211-226 (1996a).

Farkas, B., R. Singh y T. Rumsey, Modeling heat and mass transfer in immersion frying. II Model solution and verification. Journal of Food Engineering 29: 227-248 (1996b).

Freitas, D., S. Garcia, P. Prati, F. Fakhouri, F. Collares y E. Vicente, Reducing of fat uptake in cassava product during deep-fat frying. Journal of Food Engineering 94: 390-394 (2009).

González-Martínez, G., L. Ahrné, V. Gekas y I. Sjöholm, Analysis of temperature distribution in potato tissue during blanching and its effect on the absolute residual pectin methylesterase activity. Journal of FoodEngineering 65: 433-441 (2004).

Hassan, H. y H. Ramaswamy, Measurement and targeting of thermophysical properties of carrot and meat based alginate particles for thermal processing applications. Journal of Food Engineering 107: 117-126 (2011).

Huang, L. y L. Liu, Simultaneous determination of thermal conductivity and thermal diffusivity of food and agricultural materials using a transient plane-source method. Journal of Food Engineering 95: 179-185 (2009).

Incropera, F. y D. DeWitt, Fundamentos de transferencia de calor. Cuarta edición. Prentice Hall, México. Páginas 5-8 (1999).

Kassama, L. y M. Ngadi, Pore development and moisture transfer in chicken meat during deep-fat frying. Drying Technology 23: 907-923 (2005).

Kim, D., J. Lim, I. Bae, H. Lee y S. Lee, Effect of hydrocolloid coatings on the heat transfer and oil uptake during frying of potato strips. Journal of Food Engineering 102: 317-320 (2011).

Kita, A., G. Lisińska y G. Gołubowska, The effects of oils and frying temperatures on the texture and fat content of potato crisps. Food Chemistry 102: 1-5 (2007).

Mai Tran, T., X. Dong Chen y C. Southern, Reducing oil content of fried potato crisps considerably using a 'sweet' pre-treatment technique. Journal of Food Engineering 80: 719-726 (2007). 
Marcano, J., Y. La Rosa y N. Salinas, Influencia del proceso de fritura en profundidad sobre el perfil lipídico de la grasa contenida en patatas tipo "french", empleando oleína de palma. Grasas y Aceites 61 (1):24-29 (2010).

Marcotte, M., A. Taherian y Y. Karimi, Thermophysical properties of processed meat and poultry products. Journal of Food Engineering 88: 315-322 (2008).

Moreira, R., P. Da Silva y C. Gomes, The effect of a de-oiling mechanism on the production of high quality vacuum fried potato chips. Journal of Food Engineering 92: 297-304 (2009).

Mosavian, M. y V. Karizaki, Determination of mass transfer parameters during deep fat frying of rice crackers. Rice Science 19(1): 64-69 (2012).

Moyano, P. y F. Pedreschi, Kinetics of oil uptake during frying of potato slices: effect of pre-treatments. LWT 39: 285-291 (2006).

Pedreschi, F., C. Cocio, P. Moyano y E. Troncoso, Oil distribution in potato slices during frying. Journal of Food Engineering 87: 200-212 (2008).

Priya, R., R. Singhal y P. Kulkarni, Carboxymethylcellulose and hydroxypropylmethylcellulose as additives in reduction of oil content in batter based deep-fat fried boondis. Carbohydrate Polymers 29: $333-335$ (1996).

Rimac-Brnčić, S., V. Lelas, D. Rade y B. Šimundić, Decreasing of oil absorption in potato strips during deep fat frying. Journal of Food Engineering 64: 237-241 (2004).

Sablani, S., Comments on "a theoretical model to predict the formation of pores in foods during drying by $m$. S. Rahman [international journal of food properties (2003) 61-72]". International Journal of Food Properties 8: 413-414 (2005).

Sahin, S., S. Sastry y L. Bayindirli, The determination of convective heat transfer coefficient during frying. Journal of Food Engineering 39: 307-311 (1999).

Sosa-Morales, M., R. Orzuna-Espíritu y J. Vélez-Ruiz, Mass, thermal and quality aspects of deep-fat frying of pork meat. Journal of Food Engineering 77: 731-738 (2006).

Taiwo, K. y O. Baik, Effects of pre-treatments on the shrinkage and textural properties of fried sweet potatoes. LWT 40: 661-668 (2007).

Treybal, R., Operaciones de transferencia de masa. Segunda edición. Mc Graw Hill, México. Páginas 50-55 (2002).

Usawakesmanee, W., M. Chinnan, P. Wuttijumnong, A. Jangchud y N. Raksakulthai, Effect of edible coating ingredients incorporated into predusting mix on moisture content, fat content and consumer acceptability of fried breaded product. Songklanakarin J. Sci. Technol. 30(1): 25-34 (2008).

Valiente, A., Problemas de balance de materia y energía en la industria alimentaria. Segunda edición. Limusa Noriega Editores. México, 2001. Página 109 (2001).

Varela, P. y S.M. Fiszman, Hydrocolloids in fried foods. A review. Food Hydrocolloids: 1-12 (2011).

Vitrac, O., D. Dufour, G. Trystram y A. Raoult-Wack, Characterization of heat and mass transfer during deepfat frying and its effect on cassava chip quality. Journal of Food Engineering 53: 161-176 (2002).

Yamsaengsung, R. y R. Moreira, Modeling the transport phenomena and structural changes during deep fat frying Part l: model development. Journal of Food Engineering 53: 1-10 (2002).

Yildiz, A., K. Palazoglu y F. Erdogdu, Determination of heat and mass transfer parameters during frying of potato slices. Journal of Food Engineering 79:11-17 (2007).

Ziaiifar, A., N. Achir, F. Courtois, I. Trezzani y G. Trystram, Review of mechanisms, conditions, and factors involved in the oil uptake phenomenon during the deep-fat frying process. International Journal of FoodScience and Technology 43: 1410-1423 (2008). 